\title{
4
}

\section{Timing the Ischemic Stroke by Multiparametric Quantitative Magnetic Resonance Imaging}

\author{
Bryony L. McGarry ${ }^{1,2} \bullet$ Risto A. Kauppinen 3 \\ ${ }^{1}$ PRECISE4Q Predictive Modelling in Stroke, Technological University Dublin, Dublin, \\ Ireland; ${ }^{2}$ School of Psychological Science, University of Bristol, Bristol, UK and \\ ${ }^{3}$ Department of Electrical and Electronic Engineering, University of Bristol, Bristol, UK.
}

Author for correspondence: Risto A. Kauppinen, Department of Electrical and Electronic Engineering, Merchant Venturers Building, University of Bristol, Woodland Road, Bristol BS8 1UB, UK. Email:psrak@bristol.ac.uk

Doi: https://doi.org/10.36255/exonpublications.stroke.timingischemicstroke.2021

\begin{abstract}
The advent of recanalization therapies has transformed the management of acute ischemic stroke patients. The timing of symptom onset is one of the key criteria for selecting the recanalization method as pharmacological and nonpharmacological recanalization therapies are only safe when administered within strict, but evolving, time windows. Magnetic resonance imaging (MRI) reveals ischemia within minutes and estimates ischemia duration in brain parenchyma. Preclinical studies have shown that by combining diffusion and relaxometric MRI, timing ischemic strokes is possible with clinically acceptable accuracy. MRI-based stroke timing techniques have been adopted in stroke clinics to stratify patients with unknown onset time for intravenous thrombolysis, resulting in improved outcomes in clinical trials. More recent MRI approaches use absolute apparent diffusion coefficient (ADC) and $T_{2}$ relaxation time data in a user-independent manner to estimate the stroke onset time in absolute terms. The introduction of expedited MRI acquisition protocols has made MRI a fast neuro-diagnosis modality. Exploiting advanced technologies such as Magnetic Resonance Fingerprinting
\end{abstract}

In: Stroke. Dehkharghani S (Editor). Exon Publications, Brisbane, Australia. ISBN: 978-0-6450017-6-1; Doi: https://doi.org/10.36255/exonpublications.stroke.2021

Copyright: The Authors.

License: This open access article is licenced under Creative Commons Attribution-NonCommercial 4.0 International (CC BY-NC 4.0) https://creativecommons.org/licenses/by-nc/4.0/ 
(MRF), artificial intelligence (AI), and machine learning (ML) for the post-processing of MRI data, combined with fast MRI techniques, is expected to speed up the translation of objective stroke timing procedures into patient management.

Keywords: cerebral ischemia; diffusion MRI; relaxometric MRI; stroke timing; $\mathrm{T}_{2}$ relaxation time

\section{INTRODUCTION}

Imaging plays a central role in diagnosing stroke, with computerized tomography (CT) and magnetic resonance imaging (MRI) being the most commonly used in emergency departments. Verifying the presence and location of ischemia, size of the ischemic lesion, signs of brain swelling, and the presence of hemorrhage are all critical factors in the choice of treatment protocols. Tissue water is the key endogenous ingredient to CT and MRI signals. Non-contrast CT (NCCT) has a high sensitivity for hemorrhage but is less sensitive to ischemia, which is highlighted as decreased tissue density (i.e., increased water content) and takes hours to develop (1). MRI probes brain water in a much more comprehensive manner so that the evolution of ischemia-induced changes in water dynamics with concomitant tissue destruction are imaged to assess lesion characteristics beyond size, swelling, and bleeding. The introduction of recombinant tissue plasminogen activator (rtPA) to dissolve occluding blood clot(s) in the 1990s (2) meant that the duration of ischemia became one of the key clinical criteria for pharmacological patient management. In most countries, patients are eligible for intravenous (IV) rtPA if symptoms occurred within 4.5 hours of arrival to the hospital and without contraindications to the drug (3-5). Intra-arterial (IA) rtPA is considered safe within six hours of stroke onset (6), and patients with large vessel occlusion can be considered for mechanical thrombectomy (MT) within 16-24 hours $(4,7)$. As a consequence of these time windows, almost one-third of ischemic stroke patients are ineligible for reperfusion-based therapies because the time of symptom onset is unknown (8). Reasons for unknown onset time include wake-up stroke where the ischemic event occurred during sleep, the patient was found unresponsive, or symptoms were not noticed or witnessed (8). Efforts have been made to determine whether the patient may benefit from reperfusion therapies by using NCCT and MRI to estimate stroke onset time and approximate tissue viability, thus improving treatment options for patients with unknown onset time $(9,10)$.

This chapter focuses on the potential of non-contrast-enhanced MRI techniques for timing the ischemic stroke. We briefly describe the ionic and water dynamics in ischemia that are associated with MRI-detectable signals, the MRI techniques used, and the preclinical data that qualifies MRI methods for stroke timing and assessment of tissue status. Clinical applications of multiparametric MRI are reviewed, followed by a discussion on how advanced technologies such as fast MRI acquisition methods and machine learning (ML) will facilitate wider clinical use. The readers are informed that ${ }^{23} \mathrm{Na}$ MRI has also been shown to provide information about stroke onset time and tissue viability (11), which may complement the timing information obtained by the ${ }^{1} \mathrm{H}$ MRI methods covered here. 


\section{THE PATHOPHYSIOLOGY OF ISCHEMIA IN PRECLINICAL MODELS WITH REFERENCE TO MRI SIGNALS}

Preclinical studies involving animal models are essential for translational stroke research, including imaging research. Data from animal models on bioenergetics, membrane polarization, ionic distributions, and neurotransmitter activities have been crucial for understanding stroke pathophysiology and the transition to infarction $(12,13)$. Similarly, preclinical imaging studies have guided the design of MRI protocols to diagnose acute ischemia and assess tissue viability (14). The cessation of cerebral blood flow below a critical threshold (10-15ml/100g of tissue/min, but varying sigmoidally, depending upon the duration of ischemia) leads to catastrophic energy failure followed by cellular depolarizations, known as anoxic depolarization (AD) (14). AD is accompanied by cytotoxic edema (CE), which is the shift of water from the extracellular to the intracellular space. Animal data show that the diffusion-weighted (DW) MRI signal increases rapidly following energy failure, and so the ischemic region appears bright in DW images (DWI) (15). The increase in signal on DWI is a consequence of the slowed Brownian motion of tissue water, quantified by the apparent diffusion coefficient (ADC) in vivo (14). In ADC images, ischemia is represented by low intensity areas. The time-courses of ADC and AD are strongly interlinked, demonstrating that CE is one of the key causes of reduced diffusivity in ischemia (15). Preclinical data have also shown that the ADC of endogenous intracellular metabolites drops in ischemia through intracellular $\mathrm{Ca}^{2+}$-dependent mechanisms, which indicate an increase in intracellular hindrance as an additional underpinning to lowered water ADC (14).

Preclinical studies involving rodent models have established the temporal characteristics of MRI parameters caused by an ischemic stroke. Early MRI studies established that both $T_{1}$ and $T_{2}$-weighted MRI signals increase only hours after the induction of ischemia, reflecting the evolution of vasogenic edema and irreversible ischemia (16). In the hallmark paper by Mosely et al. in 1990, the DW signal increased within the first minutes of ischemia when the $\mathrm{T}_{2}$-weighted signal was still isointense (15). Fairly soon after, DW MRI entered the imaging repertoire of acute stroke clinics where MRI is available (17).

Studies have suggested that while conventional DW MRI is powerful for diagnosing ischemic stroke, it may not accurately establish the time of onset in the early stroke aftermath (17). Instead, preclinical studies have shown that in low ADC regions, indicating ischemia, absolute MRI relaxation times change almost immediately following ischemic onset $(14,18)$. Within the first moments, the $\mathrm{T}_{2}$ relaxation time shortens due to the negative blood oxygen level-dependent (BOLD), reflecting the compensatory increase of the oxygen extraction fraction (19) and a shift of extracellular water into the intracellular compartment (20). Subsequently, the $\mathrm{T}_{2}$ relaxation time shows a steady increase with time (Figure 1), initially due to CE with dissociation of intracellular supramolecular structures and eventually due to vasogenic edema $(14,19,20)$. The $\mathrm{T}_{2}$ relaxation time changes $+1.9 \mathrm{~ms} / \mathrm{h}$ during the first five hours in rat stroke with a slightly negative intercept (21). The distribution of the $\mathrm{T}_{2}$ histogram in a typical rodent stroke lesion is much narrower than human stroke lesions (21). The $\mathrm{T}_{1}$ relaxation time increases very rapidly, followed by a steady increase over time $(14,18)$. 


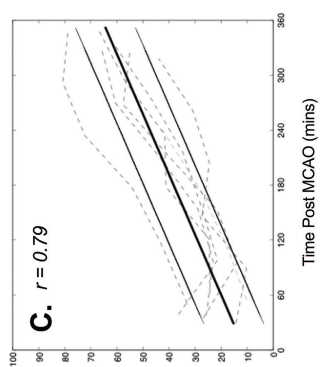

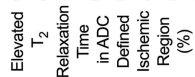
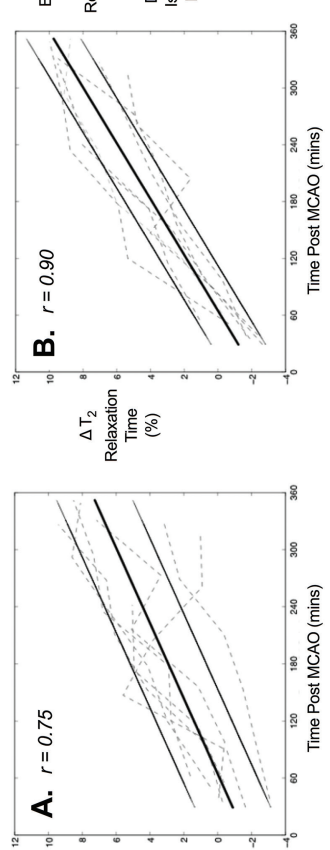

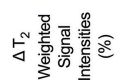
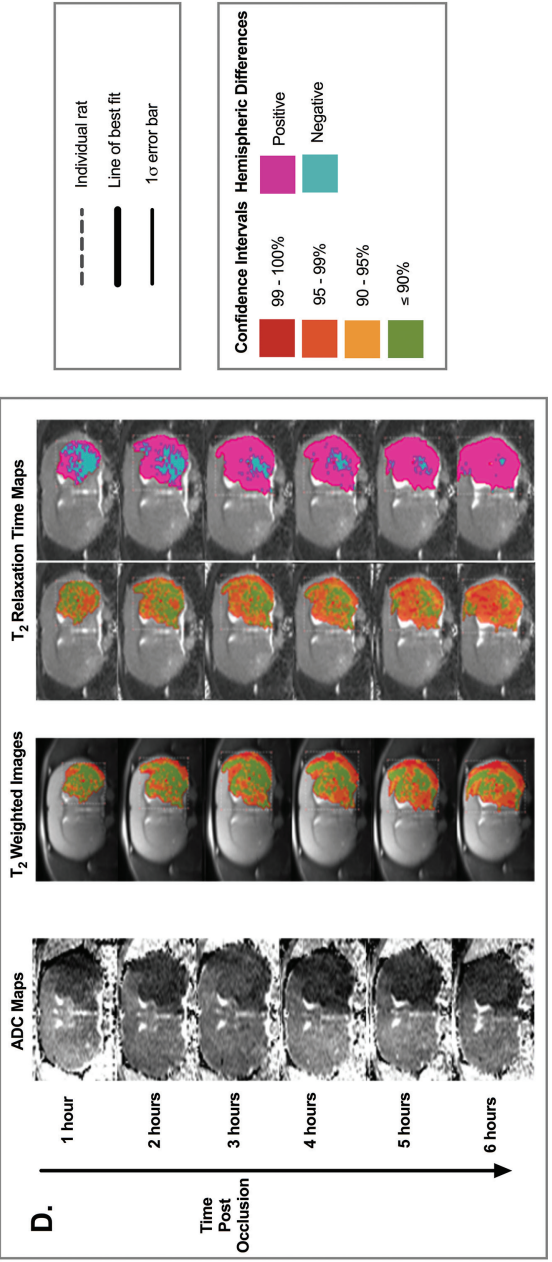

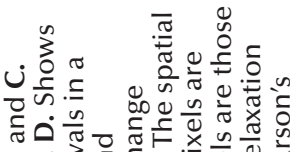
๑

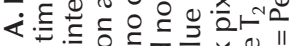

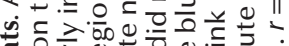

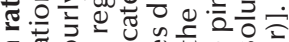

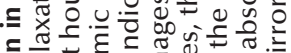

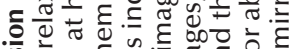

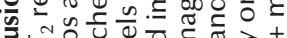

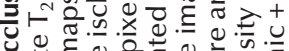

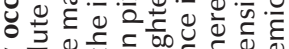

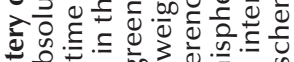

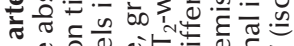

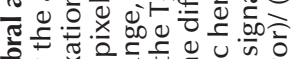

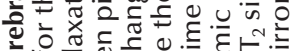

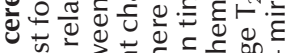

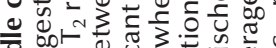
至

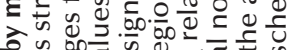

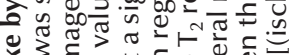

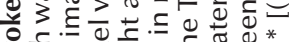

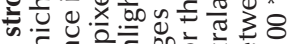
. है

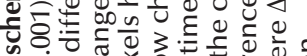

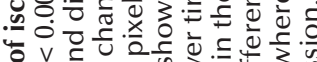

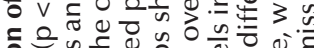

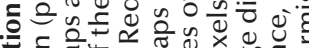

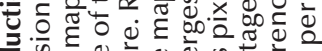
.

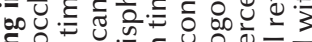

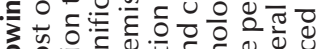

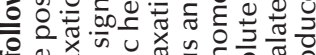

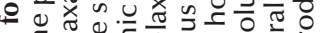

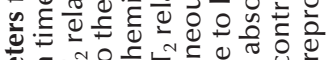

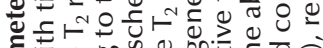

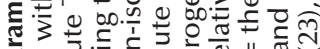

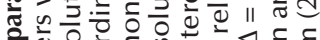

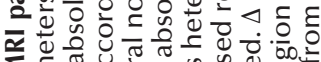

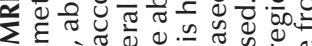

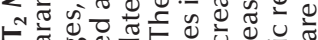

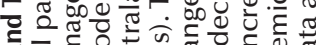
స $\bar{x}$.

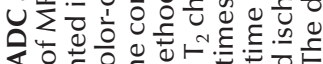
४ 을

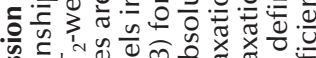
क.

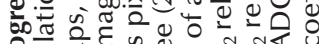
닌

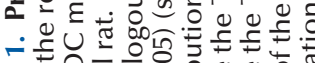

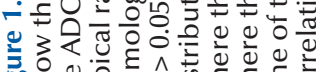

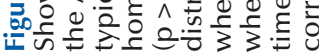


The drop in ADC within the occluded artery territory is almost instantaneous and conspicuous due to the substantial dynamic range of diffusivity, where ADC drops by $30-50 \%$ with ischemia onset (22). It is evident from these preclinical studies that the time course of changes in ADC and $T_{2}$ relaxation MRI parameters in the early hours of ischemia are both predictable and complementary, making them a suitable combination for estimating stroke onset time and evaluating tissue status. However, compared to ADC, the change in MRI signals governed by $T_{1}$ and $T_{2}$ relaxation times are much smaller and heterogeneous $(18,23,24)$. The increase in $T_{1}$ and $T_{2}$ within the first few hours is typically by a few percent $(18,23,24)$. It has been shown experimentally that these changes are difficult to identify in $T_{1}$ and $T_{2}$ weighted images during hyperacute ischemia due to the contributions of other MRI factors to the signal $(23,24)$. Instead, these changes in $T_{1}$ and $T_{2}$ relaxation times can be more explicitly and unambiguously identified and measured from parametric relaxation time maps, using robust and sophisticated methods (21).

The preclinical data suggest multiparametric MRI provides information of evolving ischemia and therefore has clinical utility. However, when exploring the potential utility of quantitative $T_{2}$ MRI for clinical use, it is vital to understand the interrelationship between ischemia pathophysiology and MRI signals and have a robust technique to quantify the subtle changes in ischemia. Thus, the CytotoxicEdema-Dissociation (CED) model was devised (20). The CED model is a mathematical model built on preclinical MRI data that incorporates the hydrodynamics in CE and subsequent dissociation of supramolecular intracellular structures with the physics of MRI measures. The CED model considers brain water in the extracellular and intracellular compartments, while the ADC and $T_{2}$ MRI signals are dealt with by the Bloch-McDonnell-Torrey regime. The CED model enables computation of the time-dependent $\mathrm{ADC}$ and $\mathrm{T}_{2}$ changes in brain parenchyma in the realistic ischemic state. The simulations suggest the rate of ischemic spread in the tissue is the crucial factor determining ADC and $\mathrm{T}_{2}$ changes. The CED model explains the time-dependency of the $\mathrm{T}_{2}$ relaxation within ADC-defined ischemic region (Figure 1B) and the heterogeneity of elevated $\mathrm{T}_{2}$ relaxation times within the ischemic region (23-25) (Figure 1D). Importantly the CED model accurately computes the time course of the $\mathrm{T}_{2}$ relaxation time using results from hydrodynamics in $\mathrm{CE}$ and the measured ADC, which validates the CED approach for clinical translation.

\section{METHODS FOR QUANTIFYING CHANGES IN THE $\mathrm{T}_{2}$ RELAXATION TIME DUE TO ISCHEMIA}

To quantify the subtle changes in $T_{2}$ relaxation due to ischemia, both userdependent and user-independent techniques have been devised in preclinical settings $(20,23,24,26,27)$ and applied to MRI data of hyperacute ischemic stroke patients $(21,28-31)$. The user-dependent mirror reference method is based on the principle that the $T_{2}$ values in the non-ischemic hemisphere indicate pre-ischemic $T_{2}$ values. The mirror reference method involves identifying the ischemic region using DWI or ADC images to create an ischemic volume of interest (VOI) (Figure 2). The ischemic VOI is then flipped across the midline 
to define the contralateral non-ischemic 'mirror reference' and loaded onto the $\mathrm{T}_{2}$-based image (Figure $2 \mathrm{C}$ ). The change in $\mathrm{T}_{2}$ due to ischemia can be approximated by calculating: (a) the difference in the average values in both VOIs in milliseconds $(26,30)$, (b) the absolute percentage change $(23,24,27)$ (Figure $1 \mathrm{~A}$ and B), or (c) by calculating the ischemic vs. non-ischemic intensity ratio $(29,31,32)$ (Figure 2D). The mirror reference method has reproducible $\mathrm{T}_{2}$ changes in rat stroke models $(23,26,27)$ and hyperacute ischemic stroke patients (28-31). It, therefore, has been proposed as a potential stroke timer, which could be used to stratify patients with unknown onset time to treatment $(23,24,26-32)$.

Due to the inherently complicated anatomy of the human brain, referencing issues are an inherent limitation of the mirror reference method when considering it for clinical use (33). Determining the subtle effects of ischemia on $T_{2}$ requires a near-perfect pre-ischemic $T_{2}$ reference $(21,33)$. The mirror reference approach is fairly reliable when applied to the rodent brain, where tissue is predominately homogeneous grey matter (34), and the $T_{2}$ distribution is narrow (33). However, in the human brain, the $\mathrm{T}_{2}$ distribution is wide across tissue types (35). Lack of perfect anatomical symmetry between hemispheres,

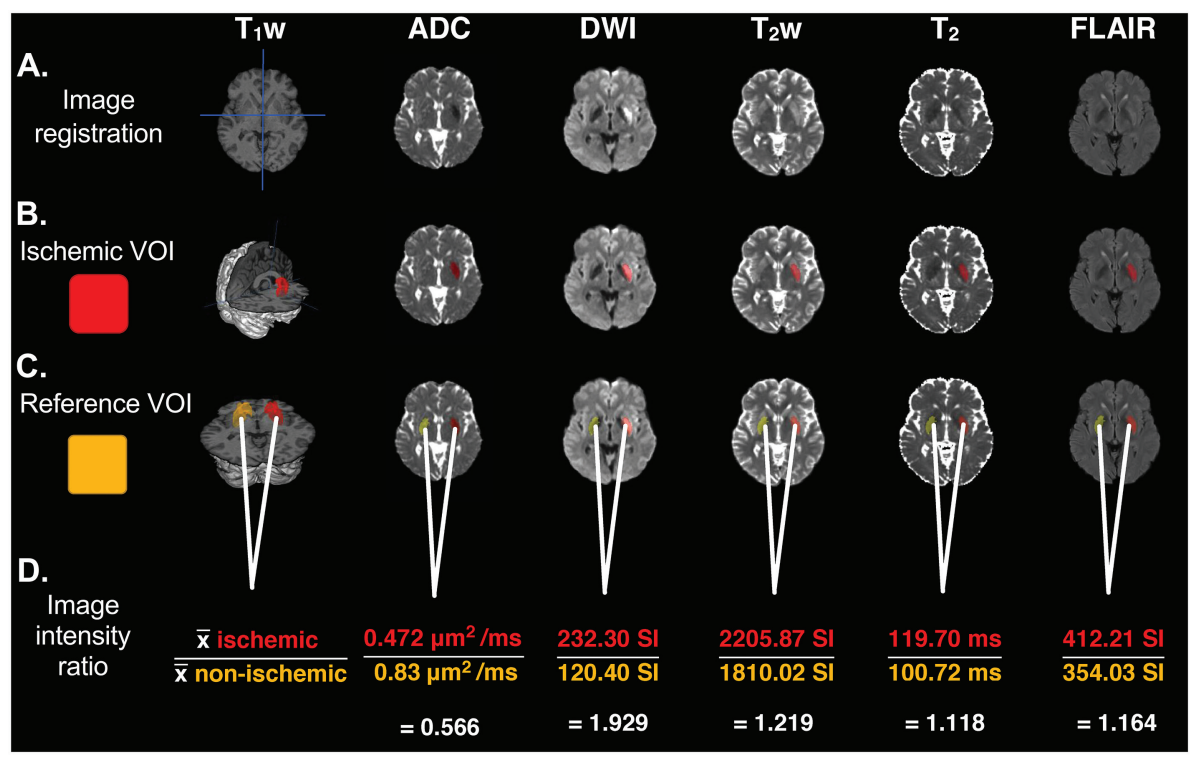

Figure 2. A mirror reference approach for quantifying changes in MRI measures in stroke. A. Images are registered to the $\mathrm{T}_{1}$ weighted image in MNI coordinate frame to correct anatomical alignment. B. ADC and absolute $\mathrm{T}_{2}$ limits are used to define the ischemic VOI. C. The mirror reference $\mathrm{VOI}$ is defined by reflecting the ischemic VOI across the midline, applying $A D C$ and $T_{2}$ limits and manually editing if necessary. $D$. The change in MR signal due to ischemia (image intensity ratio) is calculated by dividing the average of the ischemic VOI by the average of the non-ischemic VOI. Values are $\mu \mathrm{ms}^{2} / \mathrm{ms}$ for ADC, signal intensities are an arbitrary scale for weighted images, and in $\mathrm{ms}$ for absolute $\mathrm{T}_{2}$ relaxation time maps. Images are from an acute ischemic stroke patient, scanned 6 hours and 49 minutes after symptom onset; with permission from (29) and (43). 
inherently varying relaxation times across the brain, the existence of other pathologies, and $T_{2}$ anisotropy $(35,36)$ create challenges for a reliable reference region as described above. Thus, the variations in $\mathrm{T}_{2}$ across the brain are larger than the change in $\mathrm{T}_{2}$ caused by ischemia, which will inevitably introduce errors in onset time estimates (33). To overcome this problem, a reference-independent method, which exploits the distributional characteristics of $T_{2}$ within the ADC defined ischemic region, was proposed in a study on rat stroke (33).

The user-independent spherical reference method was developed using rat stroke MRI data (21) and successfully applied to MRI data from hyperacute ischemic stroke patients $(21,28)$ (Figure 3 ). The spherical reference method avoids some of the referencing issues above by achieving a more representative approximation of the pre-ischemic $T_{2}$ relaxation times using a $T_{2}$-weighted image as input. This approach takes advantage of the fact that the $\mathrm{T}_{2}$-weighted signal is unchanged or changes very little in the early moments of ischemia $(15,16)$,
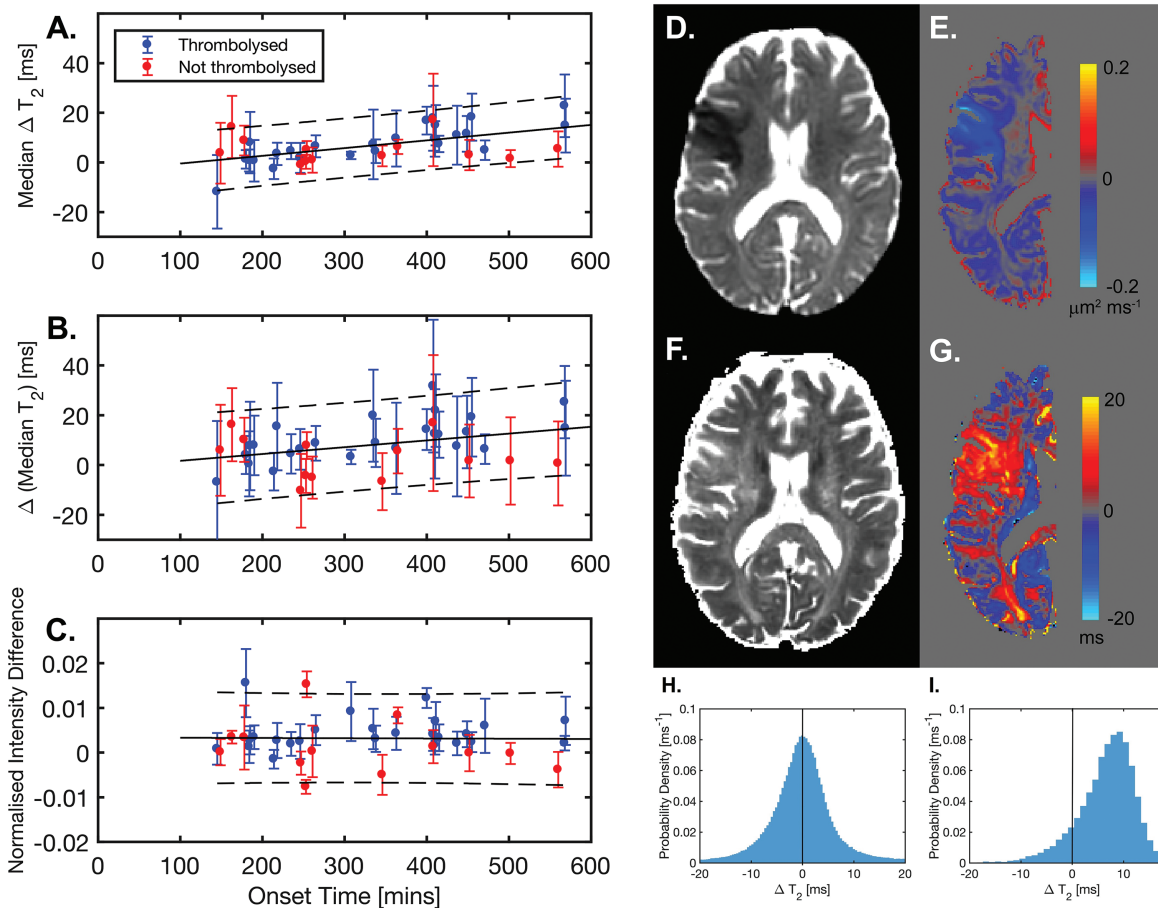
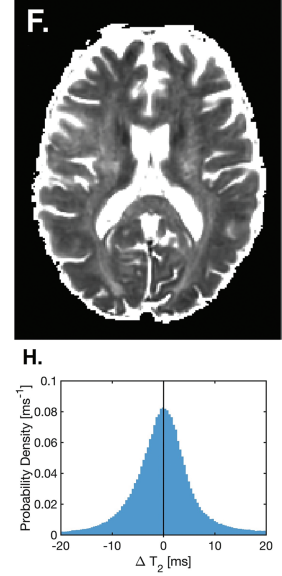

G.

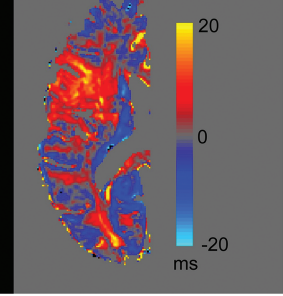

I.

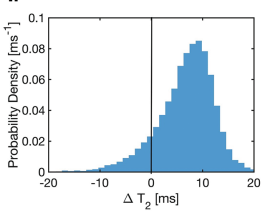

Figure 3. $A D C$ and $T_{2}$ changes in acute ischemic stroke patients by the user-independent spherical reference method. A. Median change in $T_{2}(\Delta)$ in the ADC defined ischemic regions against time from symptom onset obtained with the spherical reference method $R^{2}=0.32$, $\mathrm{p}<0.001$. B. Difference in median $\mathrm{T}_{2}$ between the lesion and contralateral mirror reference region against time from symptom onset $R^{2}=0.139, p=0.021$. C. Difference in $T_{2}$-weighted signal over time from symptom onset, where the slope is not significant from zero. D. ADC map with ischemic region visible in the right hemisphere (image left). E. Estimated change in ADC from the pre-ischemic state. F. $T_{2}$ map. G. Estimated $T_{2}$ change from the pre-ischemic state. H. $\Delta T_{2}$ distribution for normal tissue in the hemisphere of the lesion. I. $\Delta T_{2}$ distribution in the ischemic region. With permission from (21). 
thereby providing a non-ischemic signal intensity to be used in search of nonischemic reference voxels. $T_{2}$ relaxation time values are calculated in an automated fashion from the contralateral reference sphere on a voxel-by-voxel basis. For each lesion, a penalty function of finite width is computed from an individual sphere of a given radius that weights the sum of $T_{2}$ values in the voxels. This approach considers the inherent heterogeneity of lesions by computing a distribution of $T_{2}$ change caused by ischemia in the ADC defined lesion, minimizing the potential confounds caused by the anatomical differences between the lesion voxels and the contralateral hemisphere. The $T_{2}$ value due to ischemia is computed for each voxel within the ADC defined ischemic region rather than averaging across voxels as in the mirror reference approach (Figure 3).

\section{STROKE TIMING BASED ON ADC AND THE $\mathrm{T}_{2}$ MRI RELAXATION TIME IN PRECLINICAL MODELS}

Preclinical studies using the $\mathrm{T}_{2}$ relaxation to estimate stroke onset time have adopted both of the referencing methods described in this chapter. These studies have shown that linear models describe the time-dependency of $T_{2}$ changes due to ischemia well, enabling reasonably accurate estimates of onset time $(21,23,24,26,27,32)$ (Figure 1). However, the use of linear regression does not imply that $T_{2}$ relaxation has a strictly linear relationship with time, but this has served as a useful heuristic. In preclinical studies, the regression approach has yielded onset time estimates of 4.5 hours with uncertainties of \pm 35 minutes for the $\mathrm{T}_{2}$ relaxation time measured at $4.7 \mathrm{~T}$ (23) and with an error of \pm 25 minutes from $\mathrm{T}_{2}$ relaxation time data measured at 9.4T (24).

In addition to estimating time from stroke onset, rat studies have shown that the combination of ADC and the absolute $T_{2}$ relaxation time enables potentially salvageable tissue to be identified (Figure 1D). DWI does not differentiate between CE and vasogenic edema, but ADC on its own reveals CE $(14,17)$, and extensive $T_{2}$ elevation shows vasogenic edema (16). Therefore, the reduced ADC without elevated $T_{2}$ would indicate potentially salvageable tissue, an 'ADC/ $\mathrm{T}_{2}$ mismatch' (Figure 1D) $(23,24,26)$. As subtle changes are not visible on the relaxation time maps in the early hours of ischemia, methods that reveal $T_{2}$ changes have been developed in rat models $(23,24,27)$. Color-coding pixels of ADC defined ischemic regions in $T_{2}$ maps according to the significance of the $T_{2}$ change relative to the mirror reference region revealed the location of normal $\mathrm{T}_{2}$ suggesting potentially salvageable tissue and regions of elevated $T_{2}$, indicating irreversible ischemia (Figure 1D) (23). This effect is not mediated by the severity of the reduction in ADC, as the ischemic tissue is defined by a strict ADC threshold, which, as shown in preclinical $(14,18)$ and clinical $(29)$ studies, has no time dependency in hyperacute stroke. Figure $1 \mathrm{D}$ shows $\mathrm{T}_{2}$ heterogeneity within low ADC ischemic regions, indicating the uneven effects of ischemia. Interestingly, the spatial distribution of elevated $\mathrm{T}_{2}$ (Figure 1C) (23) and the volume of tissue with elevated $\mathrm{T}_{2}(24,27,32)$ within the ischemic region are also proxies for stroke onset time. 


\section{CLINICAL APPLICATION OF ADC AND T 2 MRI FOR ESTIMATING STROKE ONSET TIME AND TISSUE VIABILITY}

A large body of clinical MRI studies has treated unknown stroke onset time as a binary classification problem $(9,37)$. The ADC and $T_{2}$ MRI signals are typically used to classify a patient as within or beyond the 4.5-hour treatment window for IV rtPA. The methods are evaluated based on the overall ability to classify patients correctly, and ideally, will have high accuracy, sensitivity, and specificity for optimal treatment stratification. Stroke onset time estimators, based on diffusion and $\mathrm{T}_{2}$ signal characteristics, have been studied using a visual method, the quantitative standard mirror reference approach, and the spherical reference approaches.

The earliest work using $\mathrm{T}_{2}$ MRI to estimate ischemia duration in patients was published by Welch and colleagues (38). The more recent descendants of the $T_{2}$ approach in clinics exploit $\mathrm{T}_{2}$-FLAIR (Fluid Attenuated Inversion Recovery) MRI images (39). $\mathrm{T}_{2}$-FLAIR images are $\mathrm{T}_{2}$-weighted with attenuated contributions from 'free water' in Cerebral Spinal Fluid (CSF) and interstitial compartments. Removing these species improves the visibility of $\mathrm{T}_{2}$ changes due to stroke. The DWI/ $\mathrm{T}_{2}$-FLAIR mismatch approach combines the time-dependent sensitivities of diffusion and $T_{2}$ weighted FLAIR images to ischemia. The general concept here is that increased DWI signal (DWI positive) in the absence of increased $\mathrm{T}_{2}$-weighted FLAIR signal (FLAIR negative), termed the 'DWI/ $\mathrm{T}_{2}$-FLAIR mismatch', is suggestive of a patient likely to be within the 4.5-hour IV rtPA time window (Figure 4A). In contrast, an increased signal on both DWI and $\mathrm{T}_{2}$-FLAIR (Figure 4B) suggests the patient is beyond 4.5 hours from onset due to the presence of vasogenic edema. DWI and $\mathrm{T}_{2}$-FLAIR are analyzed using visual and semi-quantitative methods, which rely upon user input with notably low inter-rater reliability $(9,37)$.

Due to the positive results of multi-center randomized controlled trials such as WAKE-UP (39), MR-WITNESS (40), and EXTEND (41), MRI for treatment stratification of ischemic stroke patients with an unknown time of symptom onset, is recommended in clinical guidelines $(4,5)$ and is routinely used in National Institutes of Health hospitals (42). The WAKE-UP trial (39) involved visual identification of a DWI/ $T_{2}$-FLAIR mismatch (Figure 4). Patients with an unknown onset time with a visible DWI lesion and no $\mathrm{T}_{2}$-FLAIR lesion (mismatch) treated with IV rtPA showed a significantly better functional outcome than patients given placebo. The MR-WITNESS trial (40) adopted the mirror reference approach, which involved identifying the ischemic region on DWI and quantifying the $\mathrm{T}_{2}$-FLAIR signal intensities within that region (Figure 2 ). If the $\mathrm{T}_{2}$-FLAIR signal intensity ratio was less than 1.15 , the patient was treated with IV rtPA (40). It was concluded that the quantitative DWI/T 2 -FLAIR mismatch is a safe method for stratifying patients with unknown onset time to IV rtPA (40). However, it should be noted that the ratio reported is study-specific to the MRI images and cannot be used as a generic cut-off. The EXTEND trial (41) found patients with unknown onset time or with onset time between 4.5 and 9 hours with ischemic but not infarcted brain tissue (revealed by perfusion MRI), and that were treated with rtPA, had higher rates of good neurological outcome compared to those given placebo.

A well-noted problem associated with the DWI/T 2 -FLAIR mismatch approach is that it has repeatedly been shown to have low sensitivity $(9,37)$, where most patients beyond 4.5 hours from stroke onset are identified but at the expense of 


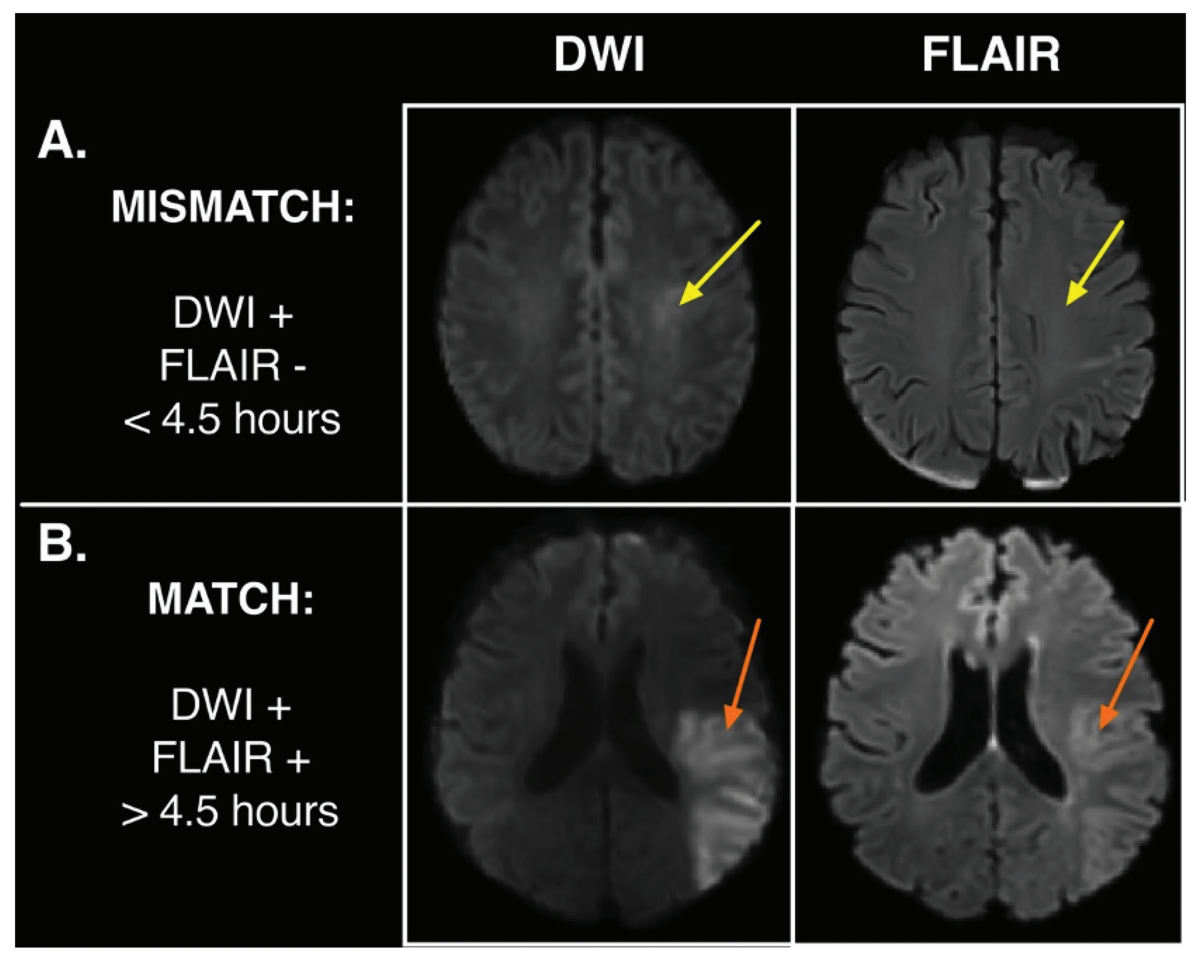

Figure 4. The DWI/T - FLAIR mismatch approach to stroke onset time estimation. A. patient is classified as within the 4.5-hour treatment window when a DWI/T 2 -FLAIR mismatch evident, where ischemic tissue is visible on DWI but not in the same region on the $T_{2}$-FLAIR image (yellow arrows). B. Patient is classified as beyond 4.5 hours if there is a match, where ischemic stissue is visible in both DWI and $\mathrm{T}_{2}$-FLAIR images (orange arrows). With permission from (43).

missing many within the 4.5-hour treatment window. The use of $\mathrm{T}_{2}$ weighted images, rather than parametric ADC and $\mathrm{T}_{2}$ maps, is the likely cause of the DWI/ $\mathrm{T}_{2}$-FLAIR mismatch's low sensitivity $(23,24,29,32)$. The MR signals of weighted images though predominantly influenced by a single parameter (i.e., diffusion or $\mathrm{T}_{2}$ relaxation), are also influenced by other factors such as proton density, $T_{1}$ relaxation, image intensity variation (the so-called bias field), and variabilities in the manufacturers' hardware and pulse sequences. Therefore, DWI and $\mathrm{T}_{2}$-FLAIR images are less 'pure' versions of diffusion and $\mathrm{T}_{2}$ than the parametric maps (43). These sources of variation result in a misrepresentation of the change in MR signals due to ischemia, adding uncertainty to onset time estimates. In support, preclinical studies have repeatedly demonstrated that $T_{2}$ relaxation times provide more accurate estimates of stroke onset time than signal intensities of $\mathrm{T}_{2}$ weighted images $(23,24,32)$. Thus, the combined $T_{2}$ weighted and DW approaches to onset time estimation are difficult to standardize.

The superior ability of $T_{2}$ relaxation times in estimating stroke onset time has also been reported in clinical studies (28-31). By adopting the mirror reference approach, a correlation using a quadratic fit between the $\mathrm{T}_{2}$ relaxation time at $1.5 \mathrm{~T}$ 
and time from symptom onset was found in hyperacute ischemic stroke patients (30). The data showed that quantifying the $T_{2}$ relaxation time was a more accurate approach than the visual $\mathrm{DWI} / \mathrm{T}_{2}$-FLAIR mismatch for distinguishing between patients scanned before and after three hours from symptom onset (30). McGarry et al. (29) extended these findings at $3 \mathrm{~T}$ to compare quantitative $\mathrm{T}_{2}$-based mirror reference methods for stroke timing in hyperacute ischemic stroke patients. Results showed that compared to $T_{2}$-weighted image intensity ratios, the absolute $T_{2}$ relaxation time ratio was the only parameter that correlated with time from symptom onset (29). The absolute $T_{2}$ ratio also had the highest accuracy for distinguishing between patients within and beyond 4.5 hours and was the only parameter with no trade-off between sensitivity and specificity (29). Therefore, unlike the visual and quantitative $\mathrm{DWI} / \mathrm{T}_{2}$-FLAIR mismatch approaches, there was a comparable classification of patients as falling within or beyond the pre-defined treatment window (29). An analysis of patients with both $\mathrm{T}_{2}$-FLAIR and $\mathrm{T}_{2}$ relaxation time images also showed that the $T_{2}$ relaxation time performed considerably better at identifying patients within 4.5 hours than the visual DWI/T 2 -FLAIR mismatch approach (29).

Methods to improve the clinical feasibility, utility, and precision of onset time estimates from $\mathrm{T}_{2}$ relaxometry have been examined (21). When applied to the absolute $T_{2}$ relaxation time in a hyperacute ischemic patient cohort, the spherical reference method was more accurate at identifying patients scanned within and beyond the 4.5-hour IV rtPA treatment window than the mirror reference approach (21). The spherical reference approach showed that at 3T, $\mathrm{T}_{2}$ changes by $+1.9 \mathrm{~ms} / \mathrm{h}$ in the ADC-defined mixed tissue type ischemic volume (21) (Figure 3). The $T_{2}$ weighted signal changes did not correlate with time from symptom onset during the first 9 hours (21). When grey and white matter components of ischemic regions were analyzed separately, the time course of $T_{2}$ relaxation times did not differ, suggesting the spherical reference method predicts onset time well, independent of tissue type and inherent tissue $T_{2}$ (28). The reported increase in $\mathrm{T}_{2}$ in the human brain is notably close to that reported in the rat brain at $9.4 \mathrm{~T}$; however, proportionally, the change at high field is approximately two-fold greater than the $T_{2}$ change at $3 T$ due to the magnetic field-dependency of $T_{2}(21,24)$.

Much of the preclinical and clinical stroke timing studies have focused on seeking linear relationships between the $\mathrm{T}_{2}$ relaxation time and time from symptom onset. However, it is recognized that the relationship between quantitative MRI parameters and time from onset is unlikely to be strictly linear due to the complex and heterogeneous nature of evolving ischemia (20,43). A preclinical MRI study highlighted this possibility by including squared and cubed terms of the original linear models' predictive variables to accommodate non-linear relationships in the $T_{2}$ data (33). Additionally, preliminary application of the preclinical study methods (33) to absolute $\mathrm{T}_{2}$ relaxation time data of hyperacute ischemic stroke patients suggested non-linearity may be specific to grey matter lesions (43). Despite the possibility of a non-linear relationship, the motivation for studying linear relationships for stroke onset time estimation is the simplicity of a linear model (43). Simplicity is key in a clinical setting, so if a linear model successfully identifies patients eligible for rtPA, it may be unnecessary to overcomplicate stroke timing methods by pursuing more complex models (43).

In addition to onset time estimation, the combination of ADC and the absolute $\mathrm{T}_{2}$ relaxation time has been shown to provide information about tissue viability in a clinical setting (44). The main benefit of quantifying ADC and $T_{2}$ is the 
opportunity to observe contributions of advancing ischemia to MRI measures that are not possible from the signal-weighted images $(20,43,44)$. One of the key challenges with DWI has been to differentiate cytotoxic and vasogenic edema's contributions to the MRI signal $(43,44)$. ADC maps are devoid of $T_{2}$ contributions from vasogenic edema. The utility of ADC and absolute $T_{2}$ images in assessing the nature of edema has been examined in chronic (45) and hyperacute ischemic stroke patients following thrombectomy (44). Within five hours post recanalization, the elevated $T_{2}$ within the ischemic region was heterogeneous (44). In 24-hour follow-up scans, the ADC had normalized, and elevated $\mathrm{T}_{2}$ predominated, suggesting irreversible damage (44). The spatial distribution of high $\mathrm{T}_{2}$ relaxation times in patients (44) is in agreement with the preclinical studies (23-25). By comparing ADC and $T_{2}$ relaxation time changes at different time points after treatment, the success of thrombectomy could be established, revealing reversible and irreversible tissue according to the $T_{2}$ relaxation time (44). Thus, the ADC and absolute $T_{2}$ data could be applied both in treatment decisions of patients with unknown onset time and monitoring response to applied therapies.

\section{EXPEDITED MRI IN HUMAN STROKE IMAGING}

One of the main concerns with using multiparametric MRI in the acute stroke setting is the time required for scan acquisitions. The duration of MRI stroke protocols is typically longer than NCCT scans, and although a 6-minute MRI protocol for acute stroke is possible (46), preparing patients for MRI requires additional time. However, since the advent of magnetic resonance fingerprinting MRF (47), and the synthetic MRI (31), quantification of absolute MRI parameters has become an order of magnitude shorter. Thus, much more MRI data can be acquired now at a similar speed as CT (47). An MRF approach was used to estimate onset time with absolute $T_{2}$ in hyperacute ischemic stroke patients (31). Referenced to a mirror volume, there was a strong correlation between the absolute $T_{2}$ and symptom onset time, whereas the correlation between $T_{2}$-FLAIR signal and onset time was weak (31). This study (31) suggests MRF is feasible for stroke timing and provides further evidence of the superior ability of absolute $T_{2}$ in onset time estimation compared to $\mathrm{T}_{2}$-FLAIR.

\section{MRI AND MACHINE LEARNING FOR THE CLINICAL ASSESSMENT OF ACUTE ISCHEMIC STROKE PATIENTS}

We are entering an era of advanced technology that will enable the development of MRI-based methods for stroke treatment stratification (48). ML is a form of artificial intelligence (AI) that involves developing algorithms to extract useful patterns from datasets to generate predictive models to be applied to new data (49). MRI-based stroke timers typically involve ML methods such as linear regression for estimating the time passed since ischemia onset and logistic regression for binary classification of patients as within or beyond a specified 
treatment time window. Although studies adopting these methods are encouraging, the clinical applicability and accuracy could be improved with other ML approaches $(43,50)$. As revealed by the CED model $(20)$ and the observed heterogeneity of $T_{2}(23,24,33,44)$, the relationship of $T_{2}$ with time is unlikely to be strictly linear (43). Consequently, using models capable of learning non-linear decision boundaries, such as support vector machines, classification trees, random forests, and discriminant analysis, may improve $\mathrm{T}_{2}$ stroke timing ability.

Another example of where ML could improve stroke timing methods is from preliminary work that classified ischemic stroke patients as within one of the three treatment time windows (IV rtPA, IA rtPA, and MT) using the absolute $\mathrm{T}_{2}$ relaxation time ratio, logistic ordinal regression, and cumulative probabilities (50). Ordinal regression models incorporating image intensity ratios from $T_{2}$ relaxation time maps or $T_{2}$ weighted images predicted the probability distribution across the predicted the probability distribution across the three treatment time windows (50). For the absolute $\mathrm{T}_{2}$ relaxation time ordinal model, accounting for the cumulative probability of a patient being within a specific time window enabled identification of patients within the middle IA rtPA treatment window, which current binary methods do not allow for (50). Identifying patients within the IA rtPA treatment window would be advantageous as it represents the transitional phase from CE to vasogenic edema, which is notoriously difficult to classify (50). The models based on $T_{2}$ weighted signal ratios could not identify patients within the IA treatment window, further supporting the superior ability of the $\mathrm{T}_{2}$ relaxation time for stroke timing (50).

Initial work adopting the end-to-end framework of deep learning (DL) has also improved MRI-based stroke timers (51). The typical linear regression and classification-based stroke timers require an a priori assumption about which factors are important in predicting onset time (i.e., absolute $T_{2}$ change). In contrast, end-to-end DL models are applied to entire images and have the freedom to learn features that the model finds useful for making accurate predictions (49). The end-to-end approach is typically used with neural networks, composed of simple computational functions that are loosely analogous to a neuron in the brain insofar as they take a set of inputs to give an output (49). A neural network has multiple interconnected neurons, allowing for the network's internal architecture to be structured in multiple ways. For image processing, convolutional neural networks (CNNs) are the exemplar of end-to-end models (49). CNNs are a way of organizing the interconnections between neurons in a network in a particularly useful manner for image processing, as they can identify local features, irrespective of location (49).

Preliminary evidence suggests stroke timing methods involving CNNs are more accurate than other ML methods (51). Using DWI and $\mathrm{T}_{2}$-FLAIR images as input, 3D CNN models enabled localization of ischemic tissue (without prior thresholding or manual delineation) and better classification of stroke patients for the 4.5-hour treatment window than the visual DWI/T ${ }_{2}$-FLAIR mismatch (51). Interestingly, the CNN model (51) had high sensitivity (0.70) and specificity (0.81), similar to that reported for the absolute $T_{2}$ relaxation time based binary classification model (sensitivity and specificity $=0.74)(29)$. Given the known limitations of $T_{2}$ weighted images and how much more representative ADC and absolute $T_{2}$ images are of stroke pathophysiology (14), DL could push the stroke timing ability of the absolute $T_{2}$ relaxation time even higher (43). The benefit of 
applying CNNs to the entire images rather than a pre-specified VOI is that physiological information, crucial to onset time estimates, is less likely to be overlooked (51). DL also affords integrating imaging and clinical data (e.g., age, blood glucose, stroke severity) termed 'multimodal fusion' (52). As well as learning hierarchies of features, the end-to-end approach can learn intramodal representations, which are not specific to imaging or clinical data but are merged representations of the two data types (52). Multimodal fusion has boosted predictive ability in other stroke research areas, such as predicting short-term outcomes (52), and therefore may also improve MRI-based stroke timing methods (43).

It is worth noting that although the application of ML has the potential to improve the predictive accuracy of MRI-based stroke timing methods, we should proceed with caution. The clinician must have confidence in the model they are using to guide critical treatment decisions, of which a key component is an understanding of how and why the model works (53). The benefit of neural networks is that they have the freedom to learn features that are useful in making predictions that the scientist may be unaware of (49). This ability has enabled powerful predictions in many medical applications (49). However, because of the extra depth, it is difficult to explain the predictive features identified by the network (53). The network could identify other phenomena or features that correlate with the output that are unrelated to the task we believe it is performing (54). Thus, before recommending clinical use, DL MRI-based models for stroke timing and tissue status evaluation must be understandable and explainable in terms of stroke pathophysiology and MR physics. Fortunately, there is extensive ongoing research focused on developing interpretability methods tailored to ML algorithms of imaging data (53). The clinician's trust could also be enhanced by including uncertainty information about the reliability of predictions made at the patient level (55). Herzog et al. (55) incorporated Bayesian uncertainty into CNNs developed for diagnosing ischemia using MRI, which showed improved prediction accuracy and higher uncertainty measures for false patient classifications enabling filtering of patients requiring closer examination. A similar component that provides uncertainty estimates associated with the predicted time window or extent of viable tissue, according to $\mathrm{T}_{2} \mathrm{MRI}$, is required.

\section{CONCLUSION}

Timing of the ischemic stroke by multiparametric MRI has already impacted treatment decisions (39), and the DWI/ $\mathrm{T}_{2}$-FLAIR mismatch is part of the routine clinical procedure for stroke treatment stratification in some centers (42). Preclinical and clinical studies suggest $\mathrm{T}_{2}$-based MRI methods for assessing hyperacute ischemic stroke patients can be improved further by quantifying the absolute $T_{2}$ relaxation time within ADC-defined ischemic regions achieved with the user-independent spherical reference method (21). We are not yet at the stage where $A D C$ and quantitative $T_{2}$ can seamlessly be used routinely in the clinic. However, Hockings et al. (56) suggest a roadmap for developing quantitative MRI-based biomarkers involving studies that focus on technical advancements such as repeatability and reproducibility to validate and standardize techniques in large multi-center studies. There is also a need to demonstrate that the methods 
are cost-effective in improving patient outcomes and public health (56). It is anticipated that advanced technologies such as MRF and ML methods will help advance the clinical feasibility and predictive ability of absolute $T_{2}$ MRI methods for treatment stratification of hyperacute ischemic stroke patients. We envisage that MRI-based stroke timers will assist in stroke care in the near future by providing the time of ischemic onset in stroke clinics. Implementation of an absolute timing regime by MRI may, in fact, push the recanalization therapy window further, giving a chance for a larger number of patients to be treated and have a more favorable outcome.

Acknowledgment: Original research at the University of Bristol was supported by The Dunhill Medical Trust (R385/1114), The Welcome Trust, and The Engineering and Physical Sciences Research Council. BLM is supported by the PRECISE4Q Predictive Modelling in Stroke project (https://precise4q.eu) funded by the European Union Horizon 2020 research and innovation programme (grant agreement No. 777107). We thank Professor John D. Kelleher (Technological University Dublin) for constructive comments.

Conflict of Interest: The authors declare no potential conflict of interest with respect to research, authorship, or publication of this chapter.

Copyright and permission statement: The authors confirm that the materials included in this chapter do not violate copyright laws. Where relevant, appropriate permissions have been obtained from the original copyright holder(s), and all original sources have been appropriately acknowledged or referenced.

\section{REFERENCES}

1. Dzialowski I, Weber J, Doerfler A, Forsting M, von Kummer R. Brain tissue water uptake after middle cerebral artery occlusion assessed with CT. J Neuroimaging. 2004;14(1):42-8. https://doi. org/10.1111/j.1552-6569.2004.tb00214.x

2. Hacke W, Kaste M, Fieschi C, von Kummer R, Davalos A, Meier D, et al. Randomised double-blind placebo-controlled trial of thrombolytic therapy with intravenous alteplase in acute ischaemic stroke (ECASS II). Lancet. 1998;352(9136):1245-51. https://doi.org/10.1016/S0140-6736(98)08020-9

3. Fiehler J, Cognard C, Gallitelli M, Jansen O, Kobayashi A, Mattle HP, et al. European recommendations on organisation of interventional care in acute stroke (EROICAS). Eur Stroke J. 2016;1(3): 155-170. https://doi.org/10.1177/2396987316659033

4. Powers WJ, Rabinstein AA, Ackerson T, Adeoye OM, Bambakidis NC, Becker K, et al. 2018 Guidelines for the early management of patients with acute ischemic stroke: A guideline for healthcare professionals from the American Heart Association/American Stroke Association. Stroke. 2018;49(3). https://doi.org/10.1161/STR.0000000000000163

5. Berge E, Whiteley W, Audebert H, Marchis GMD, Fonseca AC, Pagiglioni C, et al. European Stroke Organisation (ESO) guidelines on intravenous thrombolysis for acute ischaemic stroke. Eur Stroke J. 2021;6(1):I-LXII. https://doi.org/10.1177/2396987321989865

6. Berkhemer OA, Fransen PSS, Beumer D, van den Berg LA, Lingsma HF, Yoo AJ, et al. A randomized trial of intraarterial treatment for acute ischemic stroke. N Engl J Med. 2015;372(1):11-20. https:// doi.org/10.1056/NEJMoal411587

7. Nogueira RG, Jadhav AP, Haussen DC, Bonafe A, Budzik RF, Bhuva P, et al. Thrombectomy 6 to 24 hours after stroke with a mismatch between deficit and infarct. N Engl J Med. 2018;378(1):11-21. https://doi.org/10.1056/NEJMoal706442 
8. Dekker L, Hund H, Lemmens R, Boiten J, van den Wijngaard I. Unknown onset ischemic strokes in patients last-seen-well $>4.5 \mathrm{~h}$ : differences between wake-up and daytime-unwitnessed strokes. Acta Neurol Belg. 2017;117(3):637-42. https://doi.org/10.1007/s13760-017-0830-7

9. Etherton MR, Barreto AD, Schwamm LH, Wu O. Neuroimaging paradigms to identify patients for reperfusion therapy in stroke of unknown onset. Front Neurol. 2018;9:327. https://doi.org/10.3389/ fneur.2018.00327

10. Mair G, Alzahrani A, Lindley RI, Sandercock PAG, Wardlaw JM. Feasibility and diagnostic accuracy of using brain attenuation changes on CT to estimate time of ischemic stroke onset. Neuroradiology. 2020. https://doi.org/10.1007/s00234-020-02591-w

11. Fagan AJ. How can sodium MRI techniques help us understand acute stroke? Imaging Med. 2012;4(3):367-79. https://doi.org/10.2217/iim.12.14

12. Hossmann K-A. Pathophysiology and therapy of experimental stroke. Cell Mol Neurobiol. 2006; 26(7-8):1057-83. https://doi.org/10.1007/s10571-006-9008-1

13. Siesjö BK, Bengtsson F. Calcium Fluxes, Calcium Antagonists, and Calcium-Related Pathology in Brain Ischemia, Hypoglycemia, and Spreading Depression: A Unifying Hypothesis. J Cereb Blood Flow Metab. 1989;9(2):127-40. https://doi.org/10.1038/jcbfm.1989.20

14. Kauppinen RA. Multiparametric magnetic resonance imaging of acute experimental brain ischaemia. Prog Nucl Magn Reson Spectrosc. 2014;80:12-25. https://doi.org/10.1016/j.pnmrs.2014.05.002

15. Moseley ME, Cohen Y, Mintorovitch J, Chileuitt L, Shimizu H, Kucharczyk J, et al. Early detection of regional cerebral ischemia in cats: comparison of diffusion- and $\mathrm{T}_{2}$-weighted MRI and spectroscopy. Magn Reson Med. 1990;14(2):330-46. https://doi.org/10.1002/mrm.1910140218

16. Kato H, Kogure K, Ohtomo H, Izumiyama M, Tobita M, Matsui S, et al. Characterization of experimental ischemic brain edema utilizing proton nuclear magnetic resonance imaging. J Cereb Blood Flow Metab. 1986;6(2):212-21. https://doi.org/10.1038/jcbfm.1986.34

17. Baird AE, Warach S. Magnetic Resonance Imaging of Acute Stroke. J Cereb Blood Flow Metab. 1998;18(6):583-609. https://doi.org/10.1097/00004647-199806000-00001

18. Hoehn-Berlage M, Eis M, Back T, Kohno K, Yamashita K. Changes of relaxation times $\left(T_{1}, T_{2}\right)$ and apparent diffusion coefficient after permanent middle cerebral artery occlusion in the rat: temporal evolution, regional extent, and comparison with histology. Magn Reson Med. 1995;34(6):824-34. https://doi.org/10.1002/mrm.1910340607

19. Gröhn OHJ, Lukkarinen JA, Oja JME, van Zijl PCM, Ulatowski JA, Traystman RJ, et al. noninvasive detection of cerebral hypoperfusion and reversible ischemia from reductions in the magnetic resonance imaging relaxation time, $\mathrm{T}_{2}$. J Cereb Blood Flow Metab. 1998;18(8):911-20. https://doi. org/10.1097/00004647-199808000-00012

20. Knight MJ, McGarry BL, Rogers HJ, Jokivarsi KT, Gröhn OH, Kauppinen RA. A spatiotemporal theory for MRI $\mathrm{T}_{2}$ relaxation time and apparent diffusion coefficient in the brain during acute ischaemia: Application and validation in a rat acute stroke model. J Cereb Blood Flow Metab. 2016;36(7): 1232-43. https://doi.org/10.1177/0271678X15608394

21. Knight MJ, Damion RA, McGarry BL, Bosnell R, Jokivarsi KT, Gröhn OHJ, et al. Determining $\mathrm{T}_{2}$ relaxation time and stroke onset relationship in ischaemic stroke within apparent diffusion coefficientdefined lesions. A user-independent method for quantifying the impact of stroke in the human brain. Biomed Spectrosc Imaging. 2019;8(1-2):11-28. https://doi.org/10.3233/BSI-190185

22. Beauchamp NJ, Ulug AM, Passe TJ, van Zijl PC. MR diffusion imaging in stroke: review and controversies. Radiographics. 1998;18(5):1269-83. https://doi.org/10.1148/radiographics.18.5.9747619

23. Rogers HJ, McGarry BL, Knight MJ, Jokivarsi KT, Gröhn OHJ, Kauppinen RA. Timing the ischaemic stroke by ${ }^{1} \mathrm{H}-\mathrm{MRI}$ : improved accuracy using absolute relaxation times over signal intensities. Neuroreport. 2014;25(15):1180-5. https://doi.org/10.1097/WNR.0000000000000238

24. McGarry BL, Rogers HJ, Knight MJ, Jokivarsi KT, Sierra A, Gröhn OH, et al. Stroke onset time estimation from multispectral quantitative magnetic resonance imaging in a rat model of focal permanent cerebral ischemia. Int J Stroke. 2016;11(6):677-82. https://doi.org/10.1177/1747493016641124

25. Knight RA, Dereski MO, Helpern JA, Ordidge RJ, Chopp M. Magnetic resonance imaging assessment of evolving focal cerebral ischemia. Comparison with histopathology in rats. Stroke. 1994;25(6): 1252-61. https://doi.org/10.1161/01.STR.25.6.1252 
26. Jokivarsi KT, Hiltunen Y, Gröhn H, Tuunanen P, Gröhn OH, Kauppinen RA. Estimation of the onset time of cerebral ischemia using $\mathrm{T}_{1} \rho$ and $\mathrm{T}_{2}$ MRI in rats. Stroke. 2010;41(10):2335-40. https://doi. org/10.1161/STROKEAHA.110.587394

27. McGarry BL, Jokivarsi KT, Knight MJ, Grohn OHJ, Kauppinen RA. A magnetic resonance imaging protocol for stroke onset time estimation in permanent cerebral ischemia. JoVE. 2017;(127):e55277-e55277. https://doi.org/10.3791/55277

28. Damion RA, Knight MJ, McGarry BL, Bosnell R, Jezzard P, Harston GW, et al. Quantifying $T_{2}$ relaxation time changes within lesions defined by apparent diffusion coefficient in grey and white matter in acute stroke patients. Phys Med Biol. 2019;64(9):095016. https://doi.org/10.1088/1361-6560/ abl 1442

29. McGarry BL, Damion RA, Chew I, Knight MJ, Harston GW, Carone D, et al. A comparison of $T_{2}$ relaxation-based MRI stroke timing methods in hyperacute ischemic stroke patients: A pilot study. J Cent Nerv Syst Dis. 2020;12:1179573520943314. https://doi.org/10.1177/1179573520943314

30. Siemonsen S, Mouridsen K, Holst B, Ries T, Finsterbusch J, Thomalla G, et al. Quantitative $T_{2}$ values predict time from symptom onset in acute stroke patients. Stroke. 2009;40(5):1612-6. https://doi. org/10.1161/STROKEAHA.108.542548

31. Duchaussoy T, Budzik J-F, Norberciak L, Colas L, Pasquini M, Verclytte S. Synthetic $T_{2}$ mapping is correlated with time from stroke onset: a future tool in wake-up stroke management? 2019;29(12): 7019-7026. https://doi.org/10.1007/s00330-019-06270-0

32. McGarry BL, Rogers HJ, Knight MJ, Jokivarsi KT, Gröhn OHJ, Kauppinen RA. Determining stroke onset time using quantitative MRI: High accuracy, sensitivity and specificity obtained from magnetic resonance relaxation times. Cerebrovasc Dis Extra. 2016;6(2):60-5. https://doi. org/10.1159/000448814

33. Norton TJT, Pereyra M, Knight MJ, McGarry BM, Jokivarsi KT, Gröhn OHJ, et al. Stroke Onset Time Determination Using MRI Relaxation Times without Non-Ischaemic Reference in A Rat Stroke Model. Biomed Spectrosc Imaging. 2017;6(1-2):25-35. https://doi.org/10.3233/BSI-160155

34. Zhang K, Sejnowski TJ. A universal scaling law between gray matter and white matter of cerebral cortex. Proc Natl Acad Sci USA. 2000;97(10):5621-6. https://doi.org/10.1073/pnas.090504197

35. Wansapura JP, Holland SK, Dunn RS, Ball WS. NMR relaxation times in the human brain at 3.0 tesla. J Magn Reson Imaging. 1999;9(4):531-8. https://doi.org/10.1002/(SICI)1522-2586(199904)9: 4<531::AID-JMRI4>3.0.CO;2-L

36. Knight MJ, Wood B, Couthard E, Kauppinen R. Anisotropy of spin-echo $T_{2}$ relaxation by magnetic resonance imaging in the human brain in vivo. Biomed Spectrosc Imaging. 2015;4(3):299-310. https://doi.org/10.3233/BSI-150114

37. Wouters A, Lemmens R, Dupont P, Thijs V. Wake-up stroke and stroke of unknown onset: a critical review. Front Neurol. 2014;5:153. https://doi.org/10.3389/fneur.2014.00153

38. Welch KMA, Windham J, Knight RA, Nagesh V, Hugg JW, Jacobs M, et al. A model to predict the histopathology of human stroke using diffusion and $\mathrm{T}_{2}$-weighted magnetic resonance imaging. Stroke. 1995;26(11):1983-9. https://doi.org/10.1161/01.STR.26.11.1983

39. Thomalla G, Simonsen CZ, Boutitie F, Andersen G, Berthezene Y, Cheng B, et al. MRI-Guided thrombolysis for stroke with unknown time of onset. N Engl J Med. 2018;379(7):611-22. https://doi. org/10.1056/NEJMoal804355

40. Schwamm LH, Wu O, Song SS, Latour LL, Ford AL, Hsia AW, et al. Intravenous thrombolysis in unwitnessed stroke onset: MR WITNESS trial results. Ann Neurol. 2018;83(5):980-93. https://doi. org/10.1002/ana.25235

41. Rehani B, Ammanuel SG, Zhang Y, Smith W, Cooke DL, Hetts SW, et al. A new era of extended time window acute stroke interventions guided by imaging. Neurohospitalist. 2020 Jan;10(1):29-37. https://doi.org/10.1177/1941874419870701

42. Adil MM, Luby M, Lynch JK, Hsia AW, Kalaria CP, Nadareishvili Z, et al. Routine use of FLAIR-negative MRI in the treatment of unknown onset stroke. J Stroke Cerebrovasc Dis. 2020;29(9):105093. https:// doi.org/10.1016/j.jstrokecerebrovasdis.2020.105093

43. McGarry BL. A preclinical and clinical investigation into quantitative magnetic resonance imaging as a tool for estimating onset time in hyperacute ischaemic stroke [dissertation on the Internet for a $\mathrm{PhD}$ ]. 
Bristol, UK: University of Bristol; 2020 [cited 2021 April 08]. Available from: https://researchinformation.bris.ac.uk/en/studentTheses/a-preclinical-and-clinical-investigation-into-quantitativemagnet [Accessed on 27 April 2021]

44. Sah RG, Nobakht S, Rajashekar D, Mouches P, Forkert ND, Sitaram A, et al. Temporal evolution and spatial distribution of quantitative $\mathrm{T}_{2}$ MRI following acute ischemia reperfusion injury. Int J Stroke. 2020;15(5):495-506. https://doi.org/10.1177/1747493019895673

45. Bernarding J, Braun J, Hohmann J, Mansmann U, Hoehn-Berlage M, Stapf C, et al. Histogram-based characterization of healthy and ischemic brain tissues using multiparametric MR imaging including apparent diffusion coefficient maps and relaxometry. Magn Reson Med. 2000;43(1):52-61. https:// doi.org/10.1002/(SICI)1522-2594(200001)43:1<52::AID-MRM7>3.0.CO;2-5

46. Nael K, Khan R, Choudhary G, Meshksar A, Villablanca P, Tay J, et al. Six-minute magnetic resonance imaging protocol for evaluation of acute ischemic stroke pushing the boundaries. Stroke. 2014;45(7):1985-91. https://doi.org/10.1161/STROKEAHA.114.005305

47. Ma D, Gulani V, Seiberlich N, Liu K, Sunshine JL, Duerk JL, et al. Magnetic resonance fingerprinting. Nature. 2013;495(7440):187-92. https://doi.org/10.1038/naturel1971

48. Gulani V, Seiberlich N. Quantitative MRI: Rationale and Challenges. In: Seiberlich N, Gulani V, Calamante F, Campbell-Washburn A, Doneva M, Hu HH, et al., editors. Advances in Magnetic Resonance Technology and Applications Academic Press; 2020. p. xxxvii-li. https://doi.org/10.1016/ B978-0-12-817057-1.00001-9

49. Kelleher JD. Deep Learning. MIT Press; 2019. https://doi.org/10.7551/mitpress/11171.001.0001

50. McGarry BL, Hunter E, Damion RA, Knight MJ, Clatworthy PL, Harston GWJ, et al. Stratifying ischaemic stroke patients across 3 treatment windows using $T_{2}$ relaxation times, ordinal regression, and cumulative probabilities. Proc. Intl. Soc. Mag. Reson. Med. 2021; 29: 3265

51. Zhang H, Polson JS, Nael K, Salamon N, Yoo B, El-Saden S, et al. Intra-domain task-adaptive transfer learning to determine acute ischemic stroke onset time. ArXiv, abs/2011.03350. 2020 Nov 5.

52. Zihni E, Madai V, Khalil A, Galinovic I, Fiebach J, Kelleher J, et al. Multimodal fusion strategies for outcome prediction in stroke. HEALTHINF: Proceedings of 13th International Joint Conference on Biomedical Engineering Systems and Technologies. 2020;5:421-8. https://doi.org/ $10.5220 / 0008957304210428$

53. Zihni E, Madai VI, Livne M, Galinovic I, Khalil AA, Fiebach JB, et al. Opening the black box of artificial intelligence for clinical decision support: A study predicting stroke outcome. PLOS ONE. 2020;15(4):e0231166. https://doi.org/10.1371/journal.pone.0231166

54. Zihni E, McGarry BL, Kelleher JD. An analysis of the interpretability of neural networks trained on magnetic resonance imaging for stroke outcome prediction. Proc. Intl. Soc. Mag. Reson. Med. 2021; 29:3503

55. Herzog L, Murina E, Dürr O, Wegener S, Sick B. Integrating uncertainty in deep neural networks for MRI based stroke analysis. Med Image Anal. 2020;65:101790. https://doi.org/10.1016/j. media.2020.101790

56. Hockings P, Saeed N, Simms R, Smith N, Hall MG, Waterton JC, et al. MRI Biomarkers. In: Seiberlich N, Gulani V, Calamante F, Campbell-Washburn A, Doneva M, Hu HH, et al., editors. Advances in Magnetic Resonance Technology and Applications Academic Press; 2020. p. iii - xxxiv. https://doi. org/10.1016/B978-0-12-817057-1.00002-0 